\title{
Fusion of Electromyographic Signals with Proprioceptive Sensor Data in Myoelectric Pattern Recognition for Control of Active Transfemoral Leg Prostheses
}

\author{
A. L. Delis***, J. L. A. Carvalho*, G. A. Borges*, S.S. Rodrigues ***, I. dos Santos*, A.F. da Rocha* \\ *Department of Electrical Engineering, University of Brasília, Brasília-DF, Brazil \\ **Medical Biophysics Center, University of Oriente, Santiago de Cuba, Cuba \\ ***UnB-Gama Faculty, University of Brasília, Gama-DF, Brazil
}

\begin{abstract}
This paper presents a myoelectric knee joint angle estimation algorithm for control of active transfemoral prostheses, based on feature extraction and pattern classification. The feature extraction stage uses a combination of time domain and frequency domain methods (entropy of myoelectric signals and cepstral coefficients, respectively). Additionally, the methods are fused with data from proprioceptive sensors (gyroscopes), from which angular rate information is extracted using a Kalman filter. The algorithm uses a Levenberg-Marquardt neural network for estimating the intended knee joint angle. The proposed method is demonstrated in a normal volunteer, and the results are compared with pattern classification methods based solely on electromyographic data. The use of surface electromyographic signals and additional information related to proprioception improves the knee joint angle estimation precision and reduces estimation artifacts.
\end{abstract}

\section{METHODOLOGY}

A. Data Collection and experimental protocol

For a preliminary evaluation of the myoelectric algorithm, the following experimental protocol was designed. Two pairs of $10-\mathrm{mm} \mathrm{Ag} / \mathrm{AgCl}$ surface electrodes were placed in bipolar configuration over a pair of antagonist muscles (rectus femoris and semitendinosus muscle) of a healthy subject (Fig. 1a and $1 \mathrm{~b}$ ). These muscles correspond to the flexion and extension movements of the knee joint, respectively. The distance between the centers of the electrodes of each pair was $3-5 \mathrm{~cm}$. The reference electrodes were placed over the lateralis and medialis epicondyles bones. An electrogoniometer was placed and strapped over the external side of the leg, and the gyroscope sensors were placed over the upper and lower legs, respectively (Fig. 1c). The difference between the signals measured by the gyroscopes reflects the angular rate of the knee joint.
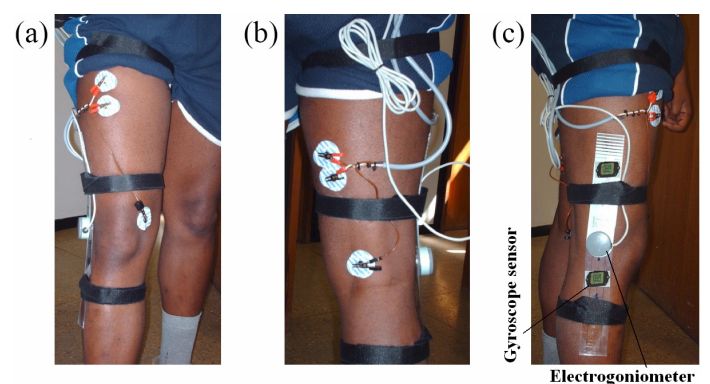

Fig. 1 : Placement of SEMG electrodes $(a, b)$, electrogoniometer and gyroscope sensors (c).

Figure 2 presents an example of SEMG signals and proprioceptive sensor data (electrogoniometer and gyroscope sensors), which were simultaneouslyacquired while the subject was walking in a constant direction and at a constant pace, for 15 seconds.

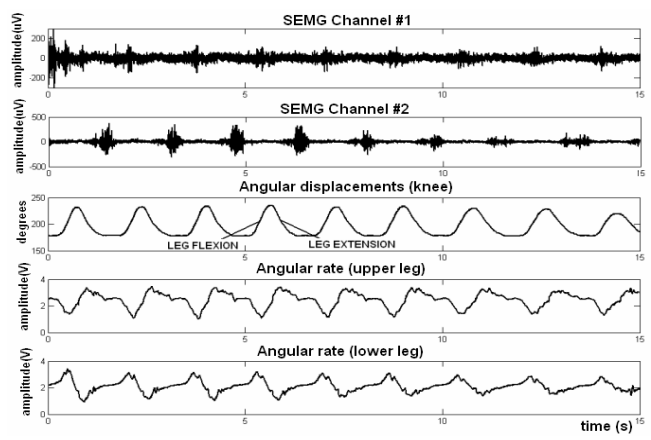

Fig. 2: Representative set of simultaneously-acquired SEMG signals (rectus femoris and semitendinosus muscles), electrogoniometer angle (knee), and gyroscope measurements (upper and lower legs).

\section{B. Data Processing}

In this work, cepstral analysis and the entropy are used for frequency and time domain SEMG signature discrimination respectively. The cepstrum of a signal is defined as the inverse Fourier transform of the logarithm of the squared magnitude of the Fourier transform of a signal, as follows [1]:

$$
c(n)=\frac{1}{N} \sum_{k=0}^{N-1} \log \left(\left.X(k)\right|^{2}\right) e^{j 2 \pi k n / N}
$$

Assuming that electromyographic signals can be approximated by a normal distribution process with zero mean, the entropy of the distribution is:

$$
\begin{aligned}
& H\left(\sigma_{i}\right)=\frac{1}{2} \log _{2}\left(2 \pi e \sigma_{i}^{2}\right) \\
& \sigma_{i}^{2}=\frac{1}{N-1} \sum_{n=1}^{N} x_{i}(n)^{2}
\end{aligned}
$$

In addition, angular rate information from the gyroscopes is used to increase angle estimation precision and reduce estimation artifacts. The goal of Kalman filters is the estimation of nonstationary signals buried in noise, by minimizing the mean squared error (i.e., recursive least squares for stochastic models) [2], according to the following linear stochastic model:

$$
\begin{aligned}
& x(k)=x(k-1)+n(k) \\
& y(k)=x(k)+v(k)
\end{aligned}
$$

This information is transferred to a three-layer LM neural network [3], with 15 nodes in the input layer, 6 nodes in the hidden layer, and 1 node in the output layer, which represents the estimated knee joint angle. The network architecture and size was empirically chosen. The true displacement angle measured with the electrogoniometer is used as training reference (Fig. 2).

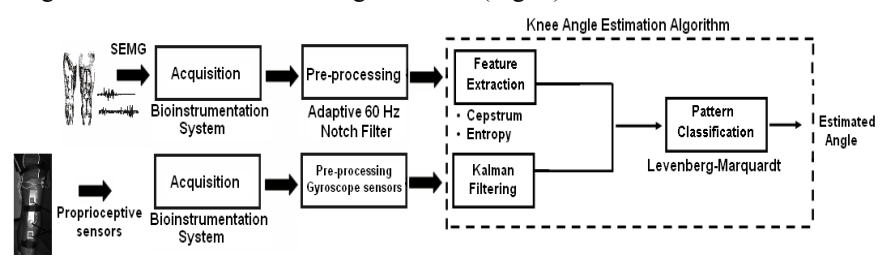

Fig. 3 : Block diagram of the proposed knee angle estimation algorithm.

\section{RESULTS AND DISCUSSION}

The results in Fig. 4 demonstrate the performance of the proposed algorithm in a representative 15 second experiment, compared with two knee angle estimation methods based solely on electromyographic data [4], [5]. The correlation between estimated and electrogoniometer-measured knee joint angles was 0.87 for the proposed method, and 0.62 and 0.81 for methods [4] and [5], respectively, in this example. The results obtained with methods [4] and [5] presented significant artifacts, which may be interpreted by the leg prosthesis as false positives, depending of their duration. These errors peaks may be due to noise in the SEMG feature space. The use of proprioceptive data considerably improved upon those methods with respect to this issue.
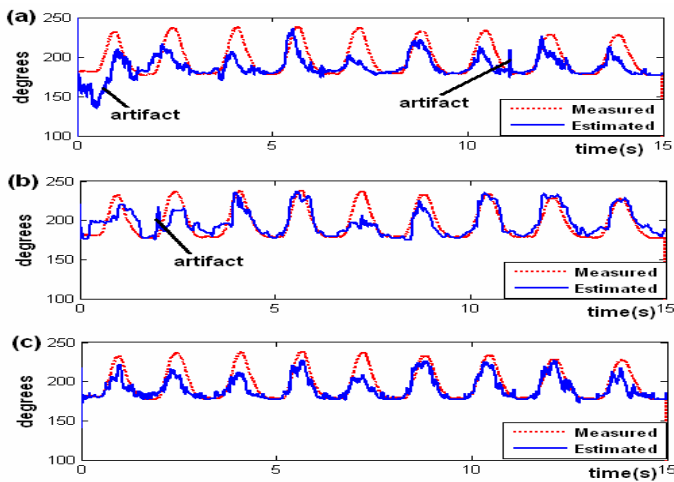

Fig. 4 : Measured and estimated knee angle displacements: (a) algorithm from ref. [4]; (b) algorithm from ref. [5]; (c) proposed algorithm.

\section{CONCLUSION}

The proposed algorithm implements data fusion of SEMG signals and proprioceptive sensor information, which improves the angle estimation precision when compared with algorithms based solely on SEMG data. The concepts used in this algorithm may be useful in the development of a control algorithm for active leg prostheses, in which signals from many different sensors may be fused and used in the conception of a movement predictive model.

\section{V.ACKNOWLEDGMENT}

This work was partially supported by CAPES and CNPq. JLA Carvalho gratefully acknowledges travel funds from Fundação de Apoio à Pesquisa do Distrito Federal (FAPDF) and from Decanato de Pesquisa e Pós-graduação da Universidade de Brasília (DPP/UnB).

\section{REFERENCES}

[1] Kang W. J., et al. IEEE Transactions on Biomedical Engineering 42:777-785.

[2] Diniz P. S. R., Kluwer Academic Publishers, 1997.

[3] Hagan M. T., et al. IEEE Trans Neural Networks 5:989-993,1994.

[4] Ferreira R.U., et al, CBB 2005.

[5] Delis A.L., et al, Biodevices 2009. 